\title{
The Femoral Prosthesis Fracture Analysis around Femur after Hip Replacement
}

\author{
Yuehao $\mathrm{Xu}^{*}$ \\ Liuyang Orthopedic Hospital, Liuyang, Hunan 410327, China
}

\begin{abstract}
Objective: To investigate the clinical effects of patients with femoral prosthesis fracture phenomena after the application of locking plate fixation for hip replacement surgery. Method: 78 cases of postoperative patients with periprosthetic femoral fracture phenomenon after hip replacement were selected and divided randomly into a control and treatment group, respectively, with an average of 39 cases per group. The control group was treated with the shape memory alloy inner ring fixing technology whereas the locking plate fixation was applied for the treatment group. Results: Fracture treatment around the femoral prosthesis in patients of treated group was better in comparison to the control group. Significantly, the treatment group revealed shorter duration of hospitalization, the recovery of limb weight-bearing capacity and the healing process as compared to the control group. Harris scores were significantly higher in the treated group in contrast to the control group. Additionally, lower adverse reaction rate was found in the treatment group as compared to the control group. Conclusion: Obviously, patient who have femoral periprosthetic fracture phenomena after receiving hip replacement were likelihood to be treated with the locking plate fixation technique.
\end{abstract}

\author{
KEYWORDS \\ Plate fixation \\ Hip replacement \\ Periprosthetic femoral fractures
}

\section{Introduction}

There are two clinical methods in order to treat the periprosthetic fracture symptoms after hip replacement which includes both non-surgical and surgical treatment, respectively. The clinical effects of locking plate fixation technique in the patients with postoperative periprosthetic femoral fracture phenomena after hip replacement treatment was investigated and followed the entire course of the report accordingly.

\section{Materials and Methods}

\subsection{Normal information}

78 cases of post-operative patients with periprosthetic femoral fracture phenomenon after hip replacement were

Copyright (C) 2016 Yuehao Xu

doi: $10.18686 /$ aem.v5i4.15

Received: September 7, 2016; Accepted: November 9, 2016;

Published online: December 30, 2015

This is an open-access article distributed under the terms of the Creative Commons Attribution Unported License (http://creativecommons.org/ licenses/by-nc/4.0/), which permits unrestricted use, distribution, and reproduction in any medium, provided the original work is properly cited.

${ }^{*}$ Corresponding author: Liuyang Orthopedic Hospital, Liuyang, Hunan 410327, China. E-mail: xuyuhao7906@sina.com selected from April 2013 to April 2015 and randomly divided into both control and treatment groups, respectively. In the control group, there were 21 males and 18 females with the age starting from 42 years old up to 86 years old. The mean age was $61.4 \pm 1.2$ years old. Fracture incidence was between 1 to 6 hours, with the onset average time of was $2.7 \pm 0.8$ hours. On the other hand, there were 22 males and 17 females in the treatment group. The patients' age was in between of 44 years old to 85 years old with the mean age $61.6 \pm 1.1$ years old. Fracture onset was between 1 to 8 hours with the onset average time was $2.9 \pm 0.7$ hours. There was no significant different between groups ( $p$ $>0.05$ ). Further comparative study can be conducted pertaining to the age, gender, disease and duration of sample.

\subsection{Method}

\subsubsection{Control group therapy}

The control group used shape memory alloy inner ring fixed technique as the treatment.

\subsubsection{Treatment group therapy}

The treatment group used the locking plate inner fixation technique as the treatment. The patients in the treated group who underwent hip replacement should receive 
anesthesia after 2 days of post operation in order to determine the type of fracture by radiological examination. During replacement, the scalpel should be incised from the outside of patients' hip under the lying position which makes about $10 \mathrm{~cm}$ in length. Then, the damaged femoral head was removed after the acetabulum was exposed and a large rotor cut the joint capsule from the center. Finally, part of the labrum was removed, and the joint replacement surgery was conducted in accordance with the hip technology-related rules. Caution should be made in order to protect the sciatic nerve during surgical procedures and the sized of acetabular cup should be selected appropriately. In addition, adjustment of the angle must be taken into account before installation procedure.

The patients in the internal fixation group should underwent laboratory examination in order to determine the type of fracture by radiological examination, in which, preoperative method should be receiving anesthesia. The patient was kept at supine position during surgical procedure. We counterpoint the position of fracture through the perspective and plate to extramedullary. Finally, the routine care and sutured incision were carried out after confirming the non-abnormalities.

\subsection{Observed indicators}

The indicators to be observed were namely, the fracture effect around the femoral prosthesis, duration of hospitalization, recovery time of limb weight-bearing capacity, healing time, margin improvement of Harris score before and after treatment, and adverse reaction rates of perioperative of two groups as a comparison.

\subsection{Treatment evaluation method}

Harris scoring system which evaluates the fracture treatment is divided into four grades i.e., excellent, good, fair, and poor. Harris score which is more than 90 points is considered as excellent. Harris score which is more than 80 points but does not reach 90 points is categorized as good. Harris score which is more than 70 points but does not reach 80 points is accounted as fair. Harris total score with less than 70 points belongs to poor $[1,2]$.

\subsection{Data analysis}

All the data were analyzed using the SPSS 17.0. We used the standard form of the mean plus or minus standard deviation $( \pm \sigma)$ in order to represent the measurement of data and the $t$ test for data analysis. $x^{2}$ test was performed in order to compare the data between the groups. It is significant different when $p<0.05$.

\section{Results}

\subsection{Fracture treatment of periprosthetic femoral}

Six patients of whom receiving the shape memory alloy circulator fixation treatment were assessed as excellent in the control group. 7 cases of patients in the control group were assessed as good with 15 cases of the patients assessed as fair and 11 patients were classified as poor. In other word, the successful rate of periprosthetic femoral fractures treatment was $71.8 \%$. In the treatment group who were treated with locking plate fixation technique, $12 \mathrm{pa}-$ tients were assessed as excellent, 16 patients were classified as good, 8 patients were assessed as fair, while 3 patients were classified as poor. In other word, the pass rate of periprosthetic femoral fractures treatment was $92.3 \%$. The treatment effect between two groups of patients was statistically significant $(p<0.05)$ as shown in Table 1.

\subsection{Duration of hospitalization, recovery time of limb weight-bearing capacity and healing time}

$20.86 \pm 3.48$ days was required to treat the patients of the control group. The capacity of limb weight-bearing returned normal after treatment was $95.18 \pm 6.39$ days while the fracture was completely healed after $116.86 \pm$ 6.52 days. $14.82 \pm 2.51$ days was needed to treat the patients of the treatment group. The capacity of limb weightbearing returned normal after treatment was $73.66 \pm 5.27$ days while the fracture was completely healed after 90.24 \pm 5.13 days. The hospitalization time, recovery time of limb weight-bearing capacity, and healing time of the two groups showed statistically significant differences $(p<0.05)$

Table 1. The treatment comparison between two groups of patients with the femoral fractures [n/(\%)].

\begin{tabular}{ccccccc}
\hline Groups & Cases $(\mathrm{n})$ & Excellent & Good & Fair & Poor & Pass rate \\
\hline The control group & 39 & $6(15.4 \%)$ & $7(17.9 \%)$ & $15(38.5 \%)$ & $11(28.2 \%)$ & $28(71.8 \%)$ \\
Treatment group & 39 & $12(30.8 \%)$ & $16(41.0 \%)$ & $8(20.5 \%)$ & $3(7.7 \%)$ & $36(92.3 \%)$ \\
$p$ & - & $<0.05$ & $<0.05$ & $<0.05$ & $<0.05$ & $<0.05$ \\
\hline
\end{tabular}

Table 2. The comparison of hospitalization time, limb weight-bearing capacity of the recovery time and healing time between two groups of patients (d).

\begin{tabular}{ccccc}
\hline Groups & Cases $(\mathrm{n})$ & Hospitalized time & Loading time & Healing Time \\
The control group & 39 & $20.86 \pm 3.48$ & $95.18 \pm 6.39$ & $116.86 \pm 6.52$ \\
Treatment group & 39 & $14.82 \pm 2.51$ & $73.66 \pm 5.27$ & $90.24 \pm 5.13$ \\
$p$ & - & $<0.05$ & $<0.05$ & $<0.05$ \\
\hline
\end{tabular}


Table 3. Improvement amplitude of Harris score between two groups before and after treatment (scores).

\begin{tabular}{ccccc}
\hline Groups & Cases $(\mathrm{n})$ & Before treatment & After treatment & $p$ \\
\hline Control group & 39 & $47.37 \pm 3.41$ & $71.64 \pm 4.52$ & $<0.05$ \\
Treatment group & 39 & $48.72 \pm 3.17$ & $84.48 \pm 4.15$ & $<0.05$ \\
$p$ & - & $>0.05$ & $<0.05$ & \\
\hline
\end{tabular}

as shown in Table 2.

\subsection{Margin improvement of Harris scores before and after treatment}

In the control group, the Harris score was $47.37 \pm 3.41$ points before treatment, while after treatment it was 71.64 \pm 4.52 points. The groups showed significant differences $(p<0.05)$. The Harris score of treated patients was 48.72 \pm 3.17 points before treatment whereas after treatment it was $84.48 \pm 4.15$ points. The groups showed significant differences $(p<0.05)$. There were no significant differences $(p>0.05)$ between the pre-treatment groups while posttreatment group revealed statistically significant difference between the groups $(p<0.05)$ (Table 3$)$.

\subsection{Adverse reactions of perioperative events}

There were eight cases in the control group of patients with adverse events in the perioperative period, with the adverse reaction rate was $20.5 \%$. There was one case of perioperative adverse reactions in treated group with the adverse reactions was $2.6 \%$. Adverse reaction rates between the two groups showed statistically significant differences $(p<0.05)$.

\section{Discussion}

Due to the presence of the prosthesis, the treatment pertaining to periprosthetic femoral fractures may be further improved especially for some elderly patients with severe osteoporosis or bone loss. If medical doctors unable to provide effective treatment, the patients will suffer from unnecessary bone pains or losses.

In this study, patients with periprosthetic femur fracture symptoms used locking plate internal fixation technique treatment after underwent hip replacement surgery. Obviously, the locking plate technology can be applied for the treatment of fractures of other parts of the body. The technology was known as closed reduction and internal fixation treatment and designed purposely for fractures, which requires minimal radical surgical operation and shows prosthesis stability [3]. After reset, the plate and bone were not attaching in a close touched so that the impact is relatively small on bone and soft tissue, as well as blood supply. Both of them have a positive role in promoting fracture healing. Fracture healing by using imaging technology can be evaluated directly through non-union of fracture phenomena findings and undergoes treatment in the early implementation of bone graft.

Chronic femoral fracture between rotors as a traumatic disease occurs mainly in the elderly patients due to their physical weakness. Therefore, the clinical effect of the treat- ment for intertrochanteric fractures for the aged patients need to adhere with the following points : (1) Shorten the preparation time before surgery on the basis that the preparation was handled perfectly; (2) Requires comprehensive laboratory tests was required before patients' surgery, such as finding on the advance treatment that could influence surgical symptoms. After the situation improved, treatment was given in accordance to the actual situation of the patient choice of treatment time; (3) Attention care may reduce remarkably the incidence of complications during bedridden, e.g., prevention of bed sores, thrombosis, and other serious infection.

Nowadays, hip replacement is a novel and arising clinical treatment approach for the treatment of aged patients pertaining to femoral rotor fracture, provided that the surgery adapted the following guidelines: (1) Age 70 and above; (2) Evans-Jensen points type III type and above; (3) Who is able to walk before fracture; (4) Who is diagnosed with serious bone lose; (5) Who is not suitable for bedridden; (6) Who is surgery-tolerance and body functioned normally; (7) Who is having less activity after surgery $[4,5]$.

Patients need to undergo anesthesia during hip replacement surgery due to the higher risk facing by the elderly patients. Epidural anesthesia was commonly used in hip replacement therapy recently. The procedure could reduce respiratory complications in the patients, however, it will increase the patients risk to death by having the cavity hematoma once they are exposed to outside. Hence, selection of the epidural anesthesia dosage in clinical applications needs to have extra caution.

\section{References}

1. Ji Q, Xin F, Liu JB. The clinical application of modified Mennen plate in femoral shaft fracture after in hip replacement. Chinese Orthopaedic Surgery. 2010;8(4):611-612.

2. Yang CC, Wei KR, Li CZ. Cyclic memory embracing fixator hip prosthesis femoral fracture. Chinese Journal of Trauma. 2009;15(6):166-167.

3. Xu CM, Chu XB, Wu HS. Fracture treatment periprosthetic femoral. Chinese Journal of Bone and Joint Injury. 2009;21(12):974.

4. Cong YJ, Liu W, Li XH. Total hip arthroplasty periprosthetic femoral fractures. Chinese Journal of Bone and Joint Surgery. 2009;2(6):434.

5. Yuan Y, Zhou H, He AY. The clinical observation of plus shank hemiarthroplasty to elderly intertrochanteric fractures. Chinese Journal of Modern Surgery. 2015;01:40-42. 